\title{
Technique of measuring the emissivity coefficient of solid materials surface
}

\author{
Irina Zharova ${ }^{1, *}$, Valery Kuznetsov ${ }^{1}$, Ksenia Perfilieva ${ }^{1}$, and Irina Zasadny ${ }^{1}$ \\ ${ }^{1}$ National Research Tomsk State University, 634050 Tomsk, Russia
}

\begin{abstract}
The option of non-stationary method of measuring the emissivity coefficient of solid materials surface is presented. Results of a model calculating experiment are given.
\end{abstract}

\section{Introduction}

Solid materials with low thermal conductivity as heat-shielding materials (HSM) are used in systems of thermal protection of elements of power plants [1-3]. In the operation of HSM at high temperatures in addition to conductive and convective component, radiation of the material surface makes a significant contribution to the total heat exchange. In this regard, there is a need to obtain data on the emission factors of HSM in the high temperatures close to the temperature of thermal destruction. In experimental studies, radiation, calorimetric and non-stationary methods are used to measure the emissivity coefficient of the surface of different materials [4, 5]. Analysis of measurement methods [5] showed that in the high temperature range it is preferable to use radiation or non-stationary methods. In $[6,7]$ a non-stationary method of determining the emissivity coefficient is proposed. The method is based on the measurement of the sample temperature during its cooling in a vacuum chamber and the interpretation of experimental data using the apparatus of inverse heat transfer problems (IHTP) [7, 8].

In this paper, we propose a modified version of the method [6], which allows to improve the accuracy and efficiency of measurements of the emissivity coefficient. Improved accuracy and efficiency of measurements is achieved through improved methods of thermophysical experiment. To reduce the measurement time, a device is proposed that provides heating of the HSM sample to the required temperature value directly in the vacuum chamber. The accuracy of the emissivity coefficient is provided by increasing the informativity of thermocouple measurements of temperature during the cooling of the sample.

\section{Calculation-experimental technique of measuring the emissivity coefficient}

\footnotetext{
* Corresponding author: zharova@niipmm.tsu.ru
} 
The scheme of the device of measuring the emissivity coefficient is shown in Fig. 1. A feature of the device is the ability to heat the sample directly in the chamber. Since the heater is placed in a vacuum chamber, it eliminates the measurement errors of the initial cooling temperature associated with the movement of the heated sample from the thermostat to the vacuum chamber.

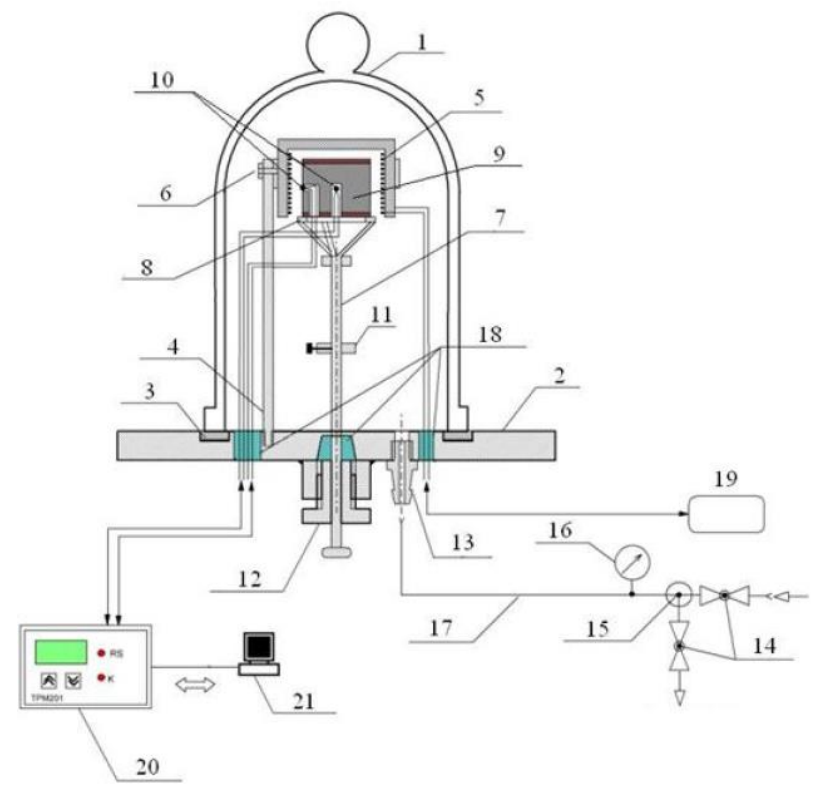

Fig. 1. The scheme of the experimental setup.

The order of measurements is as follows. In the initial position, the cap 1 with blackened walls is removed from the base 2 . The movable rod 7 is moved to the lower position until it stops 11 . The cylindrical sample 9, the end surfaces of which are covered with a foil with a high reflection coefficient, is coaxially placed on the disc 8 of the movable rod 7. The movable rod 7 is moved to the upper position to place the sample 9 inside the heater 5. To seal the chamber, clamp the seal 12 . The glass cap 1 is installed in the groove on the base 2. For complete stabilization of the residual pressure of air in the chamber produces its evacuation. Turn on the voltage source 19 and supply power to the heater 5. The temperature of the sample is controlled by thermocouples 10 , connected to the meter-regulator 20. When the required temperature of the sample is reached, the movable rod 7 is moved to the lower position and the heater 5 is switched off. Temperature measurement in the center and on the side surface of the sample during cooling is carried out without turning off the vacuum pump.

The emissivity coefficient is defined from the solution of the inverse heat conduction problem [6-8]:

$$
\frac{\partial T(r, t)}{\partial t}=a\left(\frac{\partial^{2} T(r, t)}{\partial r^{2}}+\frac{1}{r} \frac{\partial T(r, t)}{\partial r}\right)
$$

with boundary conditions and entry condition:

$$
\frac{\partial T(0, t)}{\partial r}=0, \lambda \frac{\partial T(R, t)}{\partial r}=-\varepsilon \sigma T^{4}(R, t)
$$




$$
T(r, 0)=T^{\exp }(0,0)+\left(T^{\exp }(R, 0)-T^{\exp }(0,0)\right)\left(\frac{r}{R}\right)^{2}
$$

and experimental conditions:

$$
T(0, t)=T_{1}(t), T(R, t)=T_{2}(t),
$$

where $T(r, t)$ - temperature dependence on the radial coordinate of the sample and time; $t$-time; $r$-radial coordinate of the sample; $a=\lambda /(\rho \cdot c)$-temperature conductivity coefficient of the sample; $\rho, c, \lambda$-density, specific heat, thermal conductivity of the sample; $R$ - the radius of the sample; $\sigma=5.6687 \cdot 10^{-8} \mathrm{BT} /\left(\mathrm{M}^{2} \cdot \mathrm{K}^{4}\right)-$ Stefan-Boltzmann constant; $\varepsilon$-the emissivity coefficient; $T^{\text {exp }}(0,0), T^{\text {exp }}(R, 0)$ - measured temperature values on the axis of symmetry and on the lateral surface of the sample at the start of cooling $t=0 ; T_{1}(t), T_{2}(t)$ - measured temperature dependence time on the axis of symmetry and on the lateral surface of the sample versus time during cooling.

The numerically sought value of the emissivity coefficient is determined by minimizing the functional

$$
J(\varepsilon)=\sqrt{\frac{1}{t_{k}} \sum_{i=1}^{n}\left[\left(T\left(0, t_{i}\right)-T^{\exp }\left(0, t_{i}\right)\right)^{2}+\left(T\left(R, t_{i}\right)-T^{e x p}\left(R, t_{i}\right)\right)^{2}\right]\left(t_{i}-t_{i-1}\right)},
$$

where $t_{k}$ - the cooling time of the sample; $n$-number of points used by time $(i=1,2, \ldots, n) ; T\left(0, t_{i}\right), T\left(R, t_{i}\right)$-calculated values of temperature on the axis of symmetry and on the lateral surface of the sample at the time $t_{i} ; T^{\exp }\left(0, t_{i}\right), T^{\exp }\left(R, t_{i}\right)$ - the temperature values measured at time $t_{i}$ on the symmetry axis and on the lateral surface of the sample.

\section{Results of a model calculating experiment}

The proposed experimental-calculating method was tested by numerical solution of the model problem. Characteristics of the heat-shielding material: density $\rho=2350 \mathrm{~kg} / \mathrm{m}^{3}$; specific heat $c=1000 \mathrm{~J} /(\mathrm{kg} \cdot \mathrm{K})$; coefficient of thermal conductivity $\lambda=1.4 \mathrm{~W} /(\mathrm{m} \cdot \mathrm{K})$. The radius of the cylindrical sample $R=0.015 \mathrm{~m}$, the initial temperature $T_{0}=293 \mathrm{~K}$; temperature of thermal degradation $1000 \mathrm{~K}$.

The model calculating experiment consists of two stages.

At the first stage, the temperature field in the sample is determined when heated to the temperature of thermal destruction by a constant heat flow. The temperature of the sample during the heating process is determined from the solution of the heat equation (1) with boundary conditions:

$$
\frac{\partial T(0, t)}{\partial r}=0, \lambda \frac{\partial T(R, t)}{\partial r}=q
$$

and initial condition :

$$
T(r, 0)=T_{0}=\text { const, }
$$

where $q=9.4 \cdot 10^{4} \mathrm{~W} / \mathrm{m}^{2}-$ external heat flow. 
It is obtained that on the side surface the set temperature of thermal degradation $T_{\text {term }}$ is achieved for $t_{k}=86 \mathrm{c}$. In Fig. 2 the obtained dependence of temperature on time on the axis of symmetry $T(0, t)$ and on the lateral surface $T(R, t)$ is presented numerically.

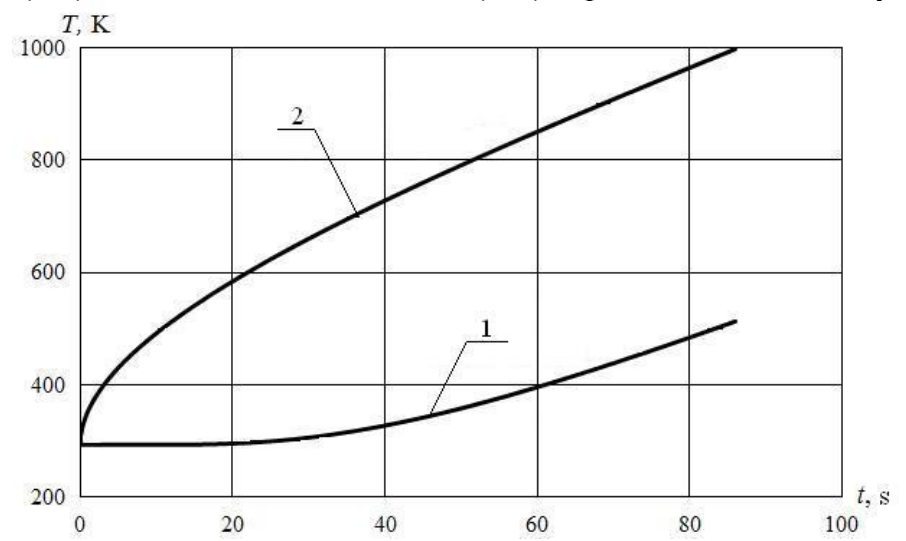

Fig. 2. Temperature dependences time on the axis of symmetry and on the lateral surface of the sample: $1-T(0, t) ; 2-T(R, t)$.

On the temperature dependences of $T(0, t)$ and $T(R, t)$ temperature distribution along the radius of the sample is determined by the equation [9]:

$$
T(r, t)=T(0, t)+(T(R, t)-T(0, t))\left(\frac{r}{R}\right) .
$$

Temperature profile along the radius of the sample at the time $t_{k}$ is shown in Fig. 3.

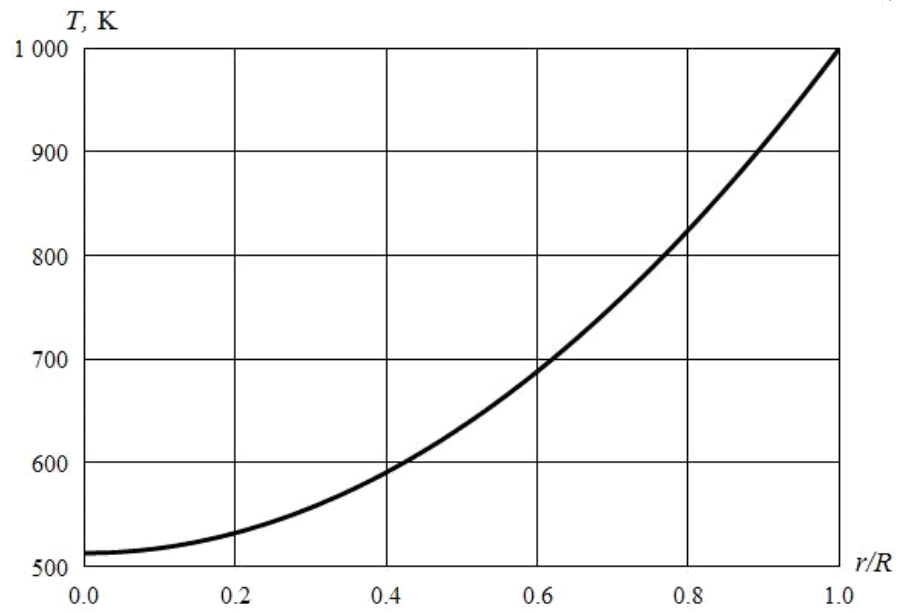

Fig. 3. Temperature profile along the radius of the sample.

At the second stage, the temperature of the sample during cooling under vacuum conditions is calculated on the basis of the solution of the direct problem of heat transfer (DPHT). The mathematical formulation of the DPHT includes equation (1) with boundary conditions (2) and initial condition (3). In the condition (3) as "experimental" temperature values are taken as $T(0,0)=513 \mathrm{~K}, T(R, 0)=1000 \mathrm{~K}$ (Fig. 2) with the introduced perturbations simulating the random error of thermocouple temperature measurements. The initial value of the emissivity coefficient is $\varepsilon_{0}=0.80$. 
The sought value of the emissivity coefficient $\varepsilon$ is obtained by solving IHTP (1)-(5). In conditions (4) "experimental" dependences $T_{1}(t), T_{2}(t)$, are defined as $T(0, t), T(R, t)$ (Fig. $4)$, that received by solving DPHT while cooling, with the introduced perturbations simulating the random error of thermocouple temperature measurements.

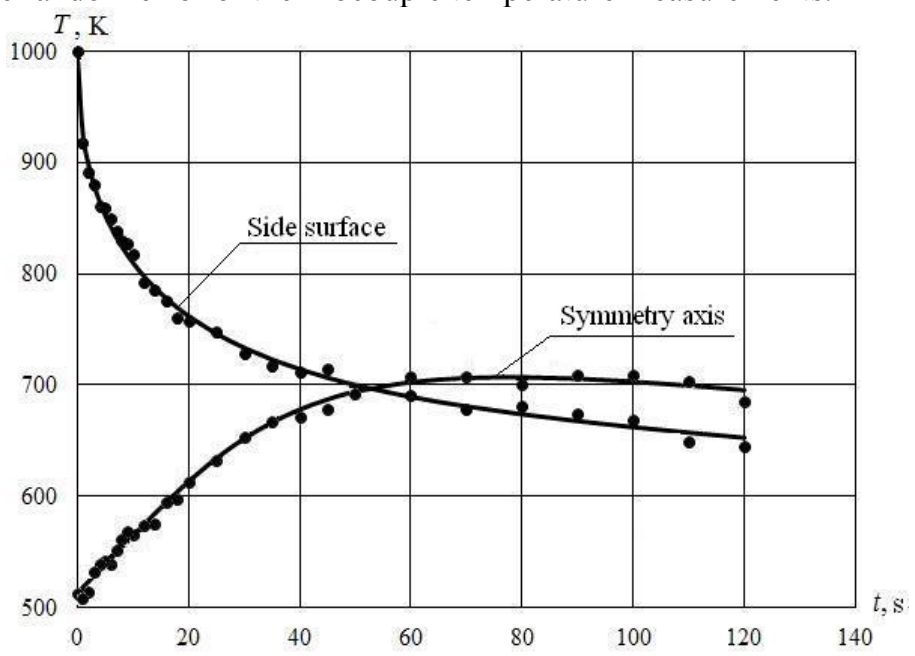

Fig. 4. Temperature dependences on the cooling time of the sample on side surface and on symmetry axis: • - "experimental"; - - calculated.

By results of calculating experiment we found the specified value $\varepsilon=0.805$, which is different from the given initial $\varepsilon_{0}$ less than $1 \%$.

\section{Conclusion}

An experimental-calculating technique implementing a modified version of the nonstationary method of measuring of the emissivity coefficient was presented. The technique is approved by carrying out a model calculating experiment. Results of calculating experiment have shown that the offered technique allows to increase the accuracy of determination the emissivity coefficient of solid materials. It is achieved by accounting of the non-uniformity of the temperature field in the sample during cooling, and also due to the increase of the informativity of the measurements due to the placement of an additional thermocouple.

This research was supported by "The Tomsk State University competitiveness improvement programe".

\section{References}

1. Yu. V. Polezhaev, F. B. Yurevich, Thermal protection (Energy, Moscow, 1976) [in Russian]

2. N. A. Rubtsov, Radiation heat transfer in continuum medium (Science, Novosibirsk, 1984) [in Russian]

3. A. G. Blokh, Yu. A. Zhuravlev, L. N. Rizhkov, Radiation heat exchange: Reference book (Energoatomizdat, Moscow, 1991) [in Russian]

4. A. Ye. Shtyndlin, Radiating properties of solid materials. Reference book (Energy, Moscow, 1974) [in Russian] 
5. V. A. Arkhipov, V. D. Gol'din, I. K. Zharova, N. I. Kurilenko, G. Ya. Mamontov, $\mathrm{T}$ and $\mathrm{A} 19$ (6), 750 (2012) [in Russian]

6. V. A. Arkhipov, V. D. Gol'din, I. K. Zharova, N. I. Kurilenko, Method for measuring the emissivity coefficient of heat-shielding materials surface Patent RU 2468360 (Int.Cl.: G01N 25/18, G01K 7/02, 2012) [in Russian]

7. A. Arkhipov, V. D. Gol'din, I. K. Zharova, N. I. Kurilenko, Instruments 2 (140), 43 (2012) [in Russian]

8. O. M. Alifanov, Inverse heat transfer problems (Mashinostroenie, Moscow, 1988) [in Russian]

9. A. V. Likov, Heat and mass transfer: Reference book (Energy, Moscow, 1971) [in Russian] 